\title{
Alessandra Mosca, Terminologie grammaticale comparative et historique du français et de l'italien : autour de l'adjectif.
}

\section{Bernard Colombat}

\section{(2) OpenEdition}

1 Journals

\section{Édition électronique}

URL : https://journals.openedition.org/dhfles/3538

DOI : $10.4000 /$ dhfles.3538

ISSN : 2221-4038

\section{Éditeur}

Société Internationale pour l'Histoire du Français Langue Étrangère ou Seconde

\section{Édition imprimée}

Date de publication : 1 décembre 2012

Pagination : p.258-262

ISSN : 0992-7654

\section{Référence électronique}

Bernard Colombat, «Alessandra mosca, Terminologie grammaticale comparative et historique du français et de l'italien : autour de l'adjectif. », Documents pour l'histoire du français langue étrangère ou seconde [En ligne], 49 | 2012, mis en ligne le 05 juillet 2016, consulté le 28 mai 2021. URL : http:// journals.openedition.org/dhfles/3538 ; DOI : https://doi.org/10.4000/dhfles.3538

Ce document a été généré automatiquement le 28 mai 2021

(c) SIHFLES 


\title{
Alessandra Mosca, Terminologie grammaticale comparative et historique du français et de l'italien : autour de l'adjectif.
}

\author{
Bernard Colombat
}

1 Thèse réalisée en co-tutelle entre l'École doctorale de linguistique française de Brescia et l'École doctorale de linguistique théorique, descriptive et automatique, université Paris Diderot, et soutenue le $1^{\mathrm{er}}$ avril 2011 à Brescia, 423 p. dont bibliographie (17 p.), annexes (67 p.), deux index (rerum et nominum). Le jury était composé de: Nadia Minerva, professeure à l'université de Catane, pré-rapportrice et rapportrice ; Bernard Colombat, professeur à l'université Paris Diderot, co-directeur, Jean-Marie Fournier, professeur à l'université Sorbonne Nouvelle Paris 3, pré-rapporteur, rapporteur et président du jury; Leandro Schena, professeur à l'université de Milan Bicocca, codirecteur. La thèse a obtenu (pour la France, l'Italie ne délivrant pas de mention) la mention « Très honorable avec les félicitations du jury à l'unanimité ».

2 La thèse porte sur la constitution de la terminologie linguistique. S'agit-il d'un sujet important? On pourrait répondre que non : depuis Saussure, l'arbitraire du signe fait que le lien entre le signifiant et le signifié est immotivé. Mais ce que les linguistes admettent pour le langage, ils le refusent le plus souvent pour la terminologie qu'ils mettent en œuvre, s'acharnant soit à motiver l'usage des termes qu'ils emploient, soit au contraire à les rendre les plus neutres et les moins chargés possible (en 1540, Scaliger proposait déjà de dénommer les cas par un numéro: premier, deuxième, troisième cas, pour éviter la charge sémantique de nominatif, génitif, etc.), soit encore créant de toutes pièces une terminologie novatrice (pensons à Beauzée ou à Damourette et Pichon). Mais la terminologie commune a un grand pouvoir de résistance à ces tentatives raisonnées, elle se constitue par à-coups échappant souvent à toute logique, comme le montre la citation de Hjelsmlev donnée en exergue (p. 3) : «On dira que la terminologie est une question de goût et ne touche pas aux réalités. 
Mais cette terminologie est malheureuse, car elle reflète un malentendu profond. » Un des résultats remarquables est que la terminologie de deux langues aussi proches dans leurs racines que le français et l'italien divergent profondément sur des sujets massifs : l'une a des déterminants (mais elle ne les a que depuis peu), l'autre a des articoli et des aggetivi determinativi; et le terme (attribut / attributo) qui qualifie le prédicat dans l'une est celui qui qualifie l'adjectif apposé au nom dans l'autre.

C'est la mise à jour des raisons historiques de ces différences qui est l'objet de cette thèse. Pour cette exploration, un gros corpus, tant du côté français que du côté italien, a été mobilisé, pour une période qui s'étend des origines des deux traditions à la terminologie officielle française de 1910 , avec, en complément, un choix d'ouvrages actuels permettant d'analyser la situation présente. L'étendue de ce corpus large, avec un peu plus de 60 ouvrages pour chacune des deux traditions (exactement 67 pour le français, 63 pour l'italien), pouvait inquiéter, dans le cadre d'un travail à réaliser en trois ou quatre ans. Alessandra Mosca l'a finalement maîtrisé, au prix d'un gros travail dont témoigne l'annexe 1 , et en sélectionnant des critères précis, comme le nombre de rééditions (p.17), critère externe s'il en est, mais qui a toute sa pertinence pour le sujet traité : étudier la terminologie sur le long terme, c'est étudier la terminologie qui «a réussi ", celle qui s'est imposée certes aux linguistes, mais aussi dans l'éducation, par le biais de l'école (de là, ces terminologies officielles dont s'est dotée la France, mais pas l'Italie, p. 25-27). Si l'accès aux textes français est facilité par Gallica, il semble plus difficile pour les textes italiens.

4 La première partie ("Terminologie grammaticale: approches synchroniques et diachroniques ", p. 123-128) pose avec clarté un certain nombre de problèmes généraux concernant la terminologie linguistique, utilisant en particulier les articles du volume que les Mémoires de la Société de linguistique de Paris avaient consacré à la publication de la journée d'études du 25 janvier 1997, journée qu'avait voulue Gilbert Lazard. La terminologie linguistique devrait et pourrait être fondée en raison, mais les choses se compliquent dès qu'on compare deux langues : que le français dise "mon chien » et l'italien "il mio cane", que l'italien utilise l'article devant le possessif (avec l'explication diachronique donnée par Rohlfs 1968, cf. p. 169) et pas le français, a évidemment des conséquences sur la dénomination des termes en jeu, puisque «les objets linguistiques, apparemment identiques, ne partagent pas toujours les mêmes propriétés» (p.60). On a là en jeu typiquement un facteur interne. Mais si attribut et attributo sont de "faux amis ", c'est pour des raisons externes : comme le dit Alessandra Mosca, « on pourrait supposer que les deux langues ont "inventé" les termes et que la relation de faux amis est une pure coïncidence, mais ce serait assez improbable " (p. 62). La suite de la première partie est consacrée à la création et à la spécialisation des termes grammaticaux dans la tradition occidentale, avec un survol, nécessairement rapide, mais efficace et bien informé, pour l'Antiquité et le Moyen Âge, suivi d'une étude plus approfondie des traditions grammaticales française et italienne. On y voit l'adjectif s'affranchir progressivement de la classe nominale.

5 La deuxième partie de la thèse ("Terminologies et objets linguistiques », p. 129-230) est consacrée à l'analyse du déterminant, un terme imposé selon Alessandra Mosca par l'analyse bloomfieldienne (determiner) et qui n'apparaît selon elle que ante litteram (p. 194 sq.) avant la période récente. Mais elle montre bien que la notion de " détermination » apparaît bien avant, et dès la logique des idées développée par PortRoyal. De fait, comme le montre la base Garnier des grammaires françaises, les termes 
en determin- se trouvent fréquemment tant dans les grammaires rédigées en latin que celles rédigées en français, mais en dépassant alors le cadre de la détermination nominale. De plus la situation était compliquée, et ce dès l'origine, puisque beaucoup de ces déterminants n'étaient pas classés de la même façon par les deux piliers de la tradition ultérieure, à savoir Donat où ils se trouvaient dans les pronoms, et Priscien où ils se trouvaient dans les noms. Dans ce dédale, Alessandra Mosca nous guide d'une main ferme, résumant systématiquement chaque développement ( $c f$. les très utiles rubriques "Pour résumer »), pointant du doigt certaines bizarreries, comme le fait qu'on dise que les déterminants ne peuvent être ni «attributs », ni « épithètes ». Elle précise: «Cet ajout peut paraitre bizarre, normalement les définitions précisent ce qu'une partie peut être et non pas le contraire; mais il est facilement justifiable par le fait que les déterminants actuels étaient classés, en français comme en italien aujourd'hui, avec les adjectifs avant les années 60. Pour justifier cette scission, les grammaires insistent sur le fait que les déterminants ne peuvent pas remplir les mêmes fonctions que les adjectifs. Et inversement, l'intégrité de la classe de l'adjectif se fonde ainsi sur la capacité de tous ses composants à remplir la fonction d'épithète ou d'attribut » (p. 174-175).

6 C'est pour les analyses de ce type que la thèse est remarquable: les scories de la tradition sont telles que même quand on réorganise la matière et qu'on redéfinit les termes, on éprouve encore le besoin de donner des informations qui correspondent en fait à la situation antérieure, et Alessandra Mosca explicite parfaitement les affirmations de ce type. Dans le dernier développement de cette deuxième partie, elle semble regretter que la grammaire scolaire italienne soit «moins institutionnalisée » (p. 226) que la grammaire française. Elle attribue cela à une dévalorisation de cette discipline due aux théories de Benedetto Croce.

7 La troisième partie, intitulée "Terminologie et histoire des idées " (p. 231-327), est consacrée à une étude des oppositions fonctionnelles attribut / épithète et attributo / predicato nominale, qui, elles, relèvent de facteurs externes à la langue et de l'histoire des idées. On y trouve des remarques fort pertinentes sur cette répartition si étonnamment différente, d'autant que l'influence de Port-Royal, pour tardive qu'elle ait été, a été grande, malgré la censure, en Italie. Alessandra Mosca pose la question de savoir pourquoi le terme prédicat ne s'est pas imposé dans la tradition française (p. 245-246), alors qu'il est « récupéré » par la tradition italienne, ainsi dans la Sintassi de Fornaciari en 1881 (p. 318), peut-être sous l'influence du prestige de la grammaire comparée allemande qui utilise prädikat (p. 325). Elle résume aussi de façon lumineuse le processus par lequel l'attributo a fini par désigner l'épithète dans la tradition italienne : «L'attributo [...] a été emprunté à la grammaire raisonnée ; ensuite, le terme a subi une translation conceptuelle tangible, en finissant par indiquer tout simplement l'adjectif. Or, quand le terme predicato s'installe pour indiquer en fait l'attribut, l'attributo est récupéré pour désigner la fonction de base de l'adjectif, celle d'épithète » (p. 322).

8 La conclusion récapitule les acquis de la thèse : deux terminologies établies sur des bases différentes, alors même que les fondements sont communs, et cela pour une part parce que les unités linguistiques ne fonctionnent pas de la même façon, et pour une autre part parce que les données théoriques de départ ont fait l'objet d'interprétations différentes. Ce qu'on cherche derrière ces terminologies, c'est évidemment une organisation conceptuelle, et on peut s'étonner avec Alessandra Mosca de voir 
considérer pronoms et déterminants comme deux classes distinctes, alors que certaines remarques des linguistes d'aujourd'hui tendraient à les rapprocher et à retrouver ainsi une unité longtemps maintenue.

9 La bibliographie est soignée et bien conçue. On trouve le corpus d'étude sous deux formes: classé par ordre chronologique dans l'introduction (p.15-22) et par ordre alphabétique dans la bibliographie (p. 333 sq.). Pour les ouvrages français, l'indication de la côte BnF ou de la disponibilité sur Gallica est fort utile.

10 La première annexe est un outil précieux. Le chercheur en histoire de la grammaire peut trouver sous la forme de deux fois deux tableaux : les catégories renfermant des déterminants dans les grammaires françaises (1), puis dans les grammaires italiennes (2) ; le traitement de l'attribut et de l'épithète dans la grammaire française (3), puis dans la grammaire italienne (4). Ce qui représente des données très précieuses, la petite rubrique « Remarques descriptives » étant utile pour situer l'ouvrage.

11 L'ensemble est complété par deux index : rerum et nominum. Le premier, qui s'imposait vu le sujet de la thèse, mais qui aurait pu s'appeler plutôt « index terminologique », est un index non extensif, ce qui est bien, mais il aurait pu être un peu plus complet (par ex. epiteto n'est pas traité que p. 322, mais déjà p. 246 ; aurait pu y figurer aussi thème/ rhème [p.245], ou encore prédicatif, proposé par Riegel comme substitut à attribut [p. 246]). L'index des noms est également sélectif et ne comporte que les noms des auteurs des corpus (y compris le corpus moderne pris comme objet de comparaison). Les textes en langue étrangère (italien, mais aussi latin) ont été systématiquement traduits. Ce travail de qualité répond, à quelques années d'intervalle, à une demande exprimée par l'organisation même du colloque Métalangage et terminologie linguistique à Grenoble en 1998 (les actes ont été publiés en 2001 chez Peeters) : celle de voir les historiens de la linguistique, et plus généralement, tous les linguistes, se pencher avec attention sur les fondements du métalangage et de la terminologie qu'ils mettent en œuvre.

\section{AUTEUR \\ BERNARD COLOMBAT}

Université Paris Diderot 